\title{
SOME WEIGHTED INEQUALITIES ON PRODUCT DOMAINS
}

\author{
HENRY LIN
}

\begin{abstract}
We extend the results of R. Fefferman [3] on the bidisc to higher product domains via induction. As an application, we extend the weighted inequality for Calderon-Zygmund operators on the bidisc to higher product domains, and we also extend the result of the Littlewood-Paley operator corresponding to the arbitrary disjoint rectangles to the weighted case.
\end{abstract}

\section{INTRODUCTION}

In [3], R. Fefferman discovered a general technique which can be used to prove weighted results in $R^{1} \times R^{1}$ (the product domain) for product weights $A_{p / 2}\left(R^{1} \times R^{1}\right)$ with $2<p<\infty$. His technique, however, explicitly used the special geometric properties of $R^{2}$. In this paper we generalize his technique to higher dimension, i.e. $R^{1} \times R^{1} \times R^{1}$ and beyond. To be more specific, we reduce Fefferman's technique to a verification of one main estimate for a linear operator $T$ :

$$
\int_{\bar{\Omega}}\left|Q_{t}(T f)(x)\right|^{2} d x \frac{d t}{t} \leq C \int_{\Omega} M\left(f^{2}\right) d x .
$$

We will explain the notation in a later section. Having this estimate, we can conclude that $T$ is a bounded map on $L^{p}(w)$ for $2<p<\infty$ and a product weight $w \in A_{p / 2}$.

Verifying the main estimate for various operators $T$ is not entirely trivial. We work on two operators; we prove the $L^{p}(w)$ boundedness for $2<p<$ $\infty, w \in A_{p / 2}$ for the product domain generalization of Calderon-Zygmund operators, and lastly we prove the following Littlewood-Paley result:

Theorem. Let $\left\{R_{l}\right\}$ be a set of disjoint rectangles with sides parallel to the coordinate axes. Let $\left\{\chi_{R_{l}}\right\}$ be their characteristic functions. Define $S_{l}(f)^{\wedge}(\xi)=$ $\chi_{R_{l}}(\xi) \widehat{f}(\xi)$. We have the operator

$$
\Delta(f)(x)=\left(\sum_{l}\left|S_{l}(f)(x)\right|^{2}\right)^{1 / 2}
$$

bounded on $L^{p}(w), 2<p<\infty$, and $w \in A_{p / 2}\left(R^{1} \times R^{1}\right)$.

Received by the editors February 9, 1988.

1980 Mathematics Subject Classification (1985 Revision). Primary 42B20; Secondary 42B25. 
In [1] Rubio de Francia first proved the above result in one dimension, then Jean-Lin Journé, in his paper [2], extended the unweighted result to higher dimensions. We are able to fill in the weighted part. For this result, many of our techniques are composites of methods developed by Rubio de Francia [1], Journé [2], and R. Fefferman [3]. We will assume that the reader is familiar with the marvelous paper of Rubio de Francia [1].

We remark that even though our product domain is labeled as $R^{1} \times \cdots \times R^{1}$, it can also be $R^{d_{1}} \times \cdots \times R^{d_{n}}$ as this requires only a change in notation.

We wish to thank the reviewer for his helpful suggestions and corrections in making this paper more intelligible.

\section{Preliminary definitions}

The proof is going to be in the language of product domains; we first review some terminology.

We say $w \in A_{p}\left(R^{1} \times \cdots \times R^{1}\right)$ if $w$ is a nonnegative measurable function on $R^{n}$, and as a function of each $x_{i}$ variable (with other variables fixed) it belongs to $A_{p}\left(R^{1}\right)$, the classical $A_{p}$ class, with its $A_{p}\left(R^{1}\right)$ norm uniformly bounded with respect to the other variables.

By a dyadic rectangle we mean a rectangle with sides parallel to the coordinate axes and whose sides are dyadic intervals. We say an interval is dyadic if it is of the form $\left[x, x+x^{k}\right), x \in 2^{k} Z$ for some integer $k$. Let $c R$ be the rectangle with the same center as the rectangle $R$ but each side is lengthened by the factor $c$.

Finally if $x \in R^{n}, t=\left(t_{1}, \ldots, t_{n}\right), t_{i}>0$, we denote by $R_{t}(x)$ the rectangle centered at $x$ with sides parallel to the axes and having side length $t_{1}, t_{2}, \ldots, t_{n}$ for each respective side. In practice, we need to associate each rectangle $R_{t}(x)$ to a dyadic rectangle. We outline the procedure. For each interval $I, 2^{k} \leq|I| \leq$ $2^{k+1}$ for an integer $k$, we associate a dyadic interval $I^{d}$ such that $\left|I^{d} \cap I\right|>\frac{1}{2}|I|$ and $\left|I^{d}\right|=2^{k}$. We can also have $2 I \supset I^{d}$ and $4 I^{d} \supset I$. Applying this to each side of $R_{t}(x)$, we can select a dyadic rectangle $R^{d}$ so that $\left|R^{d} \cap R_{t}(x)\right|>$ $\left|R_{t}(x)\right| / 2^{n}$, and $2 R_{t}(x) \supset R^{d}, 4 R^{d} \supset R_{t}(x)$.

We always let $\Omega$ be a bounded open set in $R^{n}$, and let $\sim$ be the set enlarging operator defined by

$$
\Omega_{1}=\widetilde{\Omega}=\left\{x \mid M_{s}\left(\chi_{\Omega}\right)(x)>1 / 4^{n}\right\}, \quad \Omega_{i+1}=\left(\widetilde{\Omega}_{i}\right)
$$

where $M_{s}$ is the strong maximal operator.

Next we give some appropriate extension of definitions found in [3] and [6].

Definition 1. We are in $R^{n}$. By a maximal dyadic rectangle of $\Omega$ in the $i$ th dimension we mean a dyadic rectangle $R \subset \Omega$ whose $i$ th side cannot be stretched to an even larger dyadic interval so that the resulting rectangle is still contained in $\Omega$. We call $M^{i}(\Omega)$ the collection of maximal dyadic rectangles of $\Omega$ in the $i$ th dimension. 
Definition 2. For $R \in M^{n}(\Omega), R=I_{1} \times \cdots \times I_{n}$, we let $\widehat{R}=\widehat{I}_{1} \times \cdots \times I_{n}$ where $\widehat{I}_{1}$ is the largest dyadic interval containing $I_{1}$ so that $\widehat{R} \in M^{1}(\widetilde{\Omega})$. We define $\gamma_{1}(R)=\left|\widehat{I}_{1}\right| /\left|I_{1}\right|$. Similarly we can define $\widehat{\widehat{R}}=\widehat{I}_{1} \times \widehat{I}_{2} \times I_{3} \times \cdots \times I_{n}$ in the same way, and here $\gamma_{2}(\widehat{R})=\left|\widehat{I}_{2}\right| /\left|I_{2}\right|$; similarly for $\gamma_{3}, \gamma_{4}, \ldots$ We have $\gamma_{i}=2^{m}$ for some positive integer $m$. We call these $\gamma_{i}$ "the stretching factors of $R$ over $\Omega . "$

Definition 3. Let $I$ be a dyadic interval, and let the $R^{\prime}$ be dyadic rectangles in $R^{n-1}$. We denote

$$
\begin{aligned}
E_{I} & =\bigcup\left\{R^{\prime} \subset R^{n-1} \mid I \times R^{\prime} \subset \Omega\right\}, \\
A_{I, m} & =\bigcup\left\{R^{\prime} \subset R^{n-1} \mid R=I \times R^{\prime} \in M^{n}(\Omega), \gamma_{1}(R)=2^{m}\right\} .
\end{aligned}
$$

We sometimes write $A_{I, m}$ as $A_{I}$ if no confusion exists.

The next lemma could be found in [6]. For the convenience of the readers we include a proof.

Lemma 4. Let $I$ be a dyadic interval, let $I^{\prime}$ be the unique dyadic interval containing $I$ and $\left|I^{\prime}\right|=2^{m+1}|I|$. Then $A_{I, m} \subset \widetilde{E_{i} \backslash E_{I^{\prime}}}$.

Proof. Obviously $A_{I, m} \subset E_{I}$. We show that $E_{I^{\prime}}$ does not take up too much of $A_{I, m}$. Let $R^{\prime}$ be one of the rectangles making up $A_{I, m} \cdot \gamma_{1}=2^{m}$ implies $I^{\prime} \times R^{\prime} \not \subset \widetilde{\Omega}$, that is

$$
\frac{1}{4^{n}} \geq \frac{\left|I^{\prime} \times R^{\prime} \cap \Omega\right|}{\left|I^{\prime} \times R^{\prime}\right|} \geq \frac{\left|I^{\prime} \times\left(R^{\prime} \cap E_{I^{\prime}}\right)\right|}{\left|I^{\prime} \times R^{\prime}\right|}=\frac{\left|E_{I^{\prime}} \cap R^{\prime}\right|}{\left|R^{\prime}\right|} .
$$

Therefore

$$
\left|R^{\prime} \cap\left(E_{I} \backslash E_{I^{\prime}}\right)\right| \geq\left(1-4^{-n}\right)\left|R^{\prime}\right|>\left(1 / 4^{n-1}\right)\left|R^{\prime}\right|
$$

and the result follows.

Lemma 5. Let $\Omega$ and $\widetilde{\Omega}$ be as above. Then for all $g \geq 0$ we have

$$
\int_{\widetilde{\Omega}} g d x \leq C \int_{\Omega} M_{1} \cdots M_{n}(g) d x
$$

where $M_{i}$ is the Hardy-Littlewood maximal operator acting on the ith dimension.

Proof. This is a consequence of a lemma by C. Fefferman and E. Stein [4] which states for $r>1$ and $f, g \geq 0$

$$
\int_{R^{1}}\left(M_{1} f\right)^{r} g d x \leq C_{r} \int f^{r} M_{1} g d x .
$$

We apply the above inequality in each dimension to get

$$
\int_{R^{n}}\left(M_{1} \cdots M_{n} f\right)^{r} g d x \leq C \int_{R^{n}} f^{r} M_{1} \cdots M_{n} g d x .
$$


With $f=\chi_{\Omega}$ and $\Omega^{1}=\left\{x \mid M_{1} \cdots M_{n}\left(\chi_{\Omega}\right)(x)>1 / 4^{n}\right\}$, we have from $\widetilde{\Omega} \subset \Omega^{1}$

$$
\int_{\widetilde{\Omega}} g \leq \int_{\Omega^{1}} g \leq\left(4^{r n} C\right) \int_{\Omega} M_{1} \cdots M_{n} g . \quad \text { Q. E. D. }
$$

We can now prove our version of Journé's covering lemma. The two parameter version is due to R. Fefferman [3].

Lemma (Journé's covering lemma). If $R \in M^{n}(\Omega)$, let $\gamma_{1}=\gamma_{1}(R), \gamma_{2}=$ $\gamma_{2}(\widehat{R}), \ldots$ Then for all $g \geq 0$, we have for any $\delta>0$

$$
\sum_{R \in M^{n}(\Omega)}|R| \gamma_{1}^{-\delta} \cdots \gamma_{n-1}^{-\delta} \inf _{R} g(x) \leq C_{\delta} \int_{\Omega} M g d x
$$

where $M$ is our generic notation for finitely many compositions of one-dimensional Hardy-Littlewood maximal operators.

Proof. We prove this by induction on the dimension. When $n=1$, it is obvious since the maximal dyadic intervals are disjoint. We assume the lemma for $n-1$. For $n$ we write $R=I \times R^{\prime}, x=\left(x_{1}, x^{\prime}\right), x^{\prime} \in R^{n-1}$. We are going to follow a technique of R. Fefferman [3] in sorting out $R$. We first group $R$ according to $\gamma_{1}(R)=2^{m}, 1 \leq m \leq \infty$. Having done this we further sort out $R$ according to the length $|I|$; we write $|I|=2^{i+j(m+1)}$ where $i, j$ are integers and $1 \leq i \leq(m+1)$ and group $R$ according to the double indices $i, j$. We write the left side of the formula as

$$
\sum_{m=1}^{\infty} \sum_{i=1}^{m+1} \sum_{j=-\infty}^{\infty} \sum^{\prime}|I| 2^{-\delta m} \sum_{R^{\prime} \in M^{n}\left(A_{I, m}\right)}\left|R^{\prime}\right| \gamma_{2}^{-\delta} \cdots \gamma_{n-1}^{-\delta} \inf _{x_{1} \in I} \inf _{x^{\prime} \in R^{\prime}} g\left(x_{1}, x^{\prime}\right)
$$

where $\sum^{\prime}$ extends over all $I$ with length $2^{i+j(m+1)}$.

Since $\Omega \supset I \times A_{I}$, the $\gamma_{i}, i \geq 2$, are greater than $\gamma_{i}^{\prime}$, the stretching factors of $R^{\prime}$ over $A_{I}$. Thus the last sum above is dominated by

$$
\begin{gathered}
\inf _{x_{1} \in I} \sum_{R^{\prime} \in M^{n}\left(A_{I}\right)}\left|R^{\prime}\right|\left(\gamma_{2}^{\prime} \cdots \gamma_{n-1}^{\prime}\right)^{-\delta} \inf _{x^{\prime} \in R^{\prime}} g\left(x_{1}, x^{\prime}\right) \leq C \inf _{x_{1} \in I} \int_{A_{I}} M g\left(x_{1}, x^{\prime}\right) d x^{\prime} \\
\leq C \inf _{x_{1} \in I} \int_{E_{I} \backslash E_{I^{\prime}}} M g\left(x_{1}, x^{\prime}\right) d x^{\prime}
\end{gathered}
$$

where $I^{\prime}$ is the unique dyadic interval containing $I$ with $\left|I^{\prime}\right|=2^{m+1}|I|$. We have used Lemmas 4 and 5 in the last line above. Thus the entire expression is dominated by

$$
\begin{aligned}
& \sum_{m=1}^{\infty} 2^{-\delta m} \sum_{i=1}^{m+1}\left(\sum_{j} \sum^{\prime}|I| \inf _{x_{1} \in I} \int_{E_{1} \backslash E_{I^{\prime}}} M g d x^{\prime}\right) \\
& \leq C \sum_{m} 2^{-\delta m} \sum_{i} \int_{\Omega} M g d x \\
& \leq C\left(\sum_{m=1}^{\infty} 2^{-\delta m}(m+1)\right) \int_{\Omega} M g d x
\end{aligned}
$$


The first inequality holds because the sets $\left\{I \times E_{I} \backslash E_{I^{\prime}}\right\}$ as indexed by $j$ (which appeared in $|I|$ and $\left.\left|I^{\prime}\right|\right)$ are disjoint. This gives the covering lemma.

\section{THE GENERAL PROCEDURE}

We outline here a procedure which can be in general used to yield the weighted norm result for $2<p<\infty$, and a weight $w \in A_{p / 2}$ for product domains. This is taken from R. Fefferman [3] with minor adjustments.

We begin by assuming a main estimate. In practice this is the step that requires checking. The proof of this estimate for certain operators $T$ will be carried out in later sections.

Assumption (the main estimate). Let $\Omega$ be open and bounded in $R^{n}$. Let $t=\left(t_{1}, \ldots, t_{n}\right), t_{i}>0$, and let $Q_{t}$ be the convolution operator with $\prod_{i=1}^{n}\left(1 / t_{i}\right) \psi\left(x_{i} / t_{i}\right)$ where $\int \psi=0$ and $\psi$ is supported on $[-1 / 2,1 / 2]$. Let $R_{t}(x)$ be a rectangle centered at $x$ with $i$ th side having length $t_{i}$. Define $\bar{\Omega}=\left\{(x, t) \mid R_{t}(x) \subset \Omega\right\}$. Then we have

$$
\int_{\bar{\Omega}}\left|Q_{t}(T f)\right|^{2} d x \frac{d t}{t} \leq C \int_{\Omega} M\left(f^{2}\right) d x
$$

where $d x=d x_{1} \cdots d x_{n}, d t / t=d t_{1} / t^{\prime} \cdots d t_{n} / t_{n}$, and $M$ is finitely many compositions of one dimensional Hardy-Littlewood maximal operators.

Theorem. If the main estimate holds, then the linear operator $T$ is bounded on $L^{p}(w)$, for $2<p<\infty$ and $w \in A_{p / 2}\left(R^{1} \times \cdots \times R^{1}\right)$.

Proof of the theorem. We use the product Lusin area integral

$$
S(f)(x)=\left(\int_{\Gamma(x)}\left|Q_{t} f\right|^{2} d y \frac{d t}{t^{n+1}}\right)^{1 / 2}
$$

where $\Gamma(x)$ is the tensor product of the cones $\left\{\left(y_{i}, t_{i}\right)|| y_{i}-x_{i} \mid<t_{i}\right\}$. It is known that $\|S f\|_{L^{p}(w)}$ is equivalent to $\|f\|_{L^{p}(w)}$ for this range of $p$. Thus for $2<p<\infty, w \in A_{p / 2}$,

$$
\|T(f)\|_{L^{p}(w)} \leq C\|S(T f)\|_{L^{p}(w)} .
$$

We need to show that for $\phi \in C_{c}^{\infty}\left(R^{n}\right),\|\phi\|_{L^{(p / 2)^{\prime}}(w)} \leq 1$,

$$
\int\{S(T f)\}^{2} \phi w d x \leq C\left(\int f^{p} w d x\right)^{2 / p} .
$$

Explicitly writing out $S(T f)^{2}$ and interchanging the integrals, we get

$$
\int S(T f)^{2} \phi w d x=\int_{\left(R_{+}^{2}\right)^{n}}\left|Q_{t}(T f)(y)\right|^{2}\left(\frac{1}{\left|R_{t}(y)\right|} \int_{R_{t}(y)} \phi w d x\right) d y \frac{d t}{t} .
$$

Let us define

$$
\begin{aligned}
O_{k} & =\left\{x \mid M_{s}(\phi w)(x)>2^{k}\right\}, \\
A_{k} & =\left\{(y, t) \mid R_{t}(y) \subset O_{k}, R_{t}(y) \not \subset O_{k+1}\right\} .
\end{aligned}
$$


We remark that since $\phi$ has compact support, $O_{k}$ is always open and bounded. The expression in (1) is dominated by

$$
\sum_{k=-\infty}^{+\infty} 2^{k+1} \int_{A_{k}}\left|Q_{t}(T f)(y)\right|^{2} d y \frac{d t}{t}
$$

Now $A_{k} \subset \bar{O}_{k}$, so we can replace $A_{k}$ by $\bar{O}_{k}$ and apply the main estimate. (2) then is bounded by

$$
\sum_{k=-\infty}^{\infty} 2^{k+1} \int_{O_{k}} M\left(f^{2}\right) d x
$$

And this is equivalent to

$$
\int M\left(f^{2}\right) M_{s}(\phi w) d x
$$

which means (1) becomes

$$
\int S(T f)^{2} \phi w d x \leq C \int M\left(f^{2}\right) M_{s}(\phi w) d x .
$$

Finally, we get the weighted inequality by multiplying the left-hand side above by $w^{2 / p} / w^{2 / p}$ and applying Hölder's inequality with exponents $p / 2,(p / 2)^{\prime}$. The left side is dominated by

$$
\left(\int M\left(f^{2}\right)^{p / 2} w d x\right)^{2 / p} \cdot\left(\int M_{s}(\phi w)^{(p / 2)^{\prime}} w^{1-(p / 2)^{\prime}} d x\right)^{1 /(p / 2)^{\prime}} .
$$

From the fact that $M$ and $M_{s}$ are bounded with respect to weights $w$ and $w^{1-\left(p / 2^{\prime}\right)} \in A_{(p / 2)^{\prime}}$, we have

$$
\int S(T f)^{2} \phi w d x \leq C\left(\int f^{p} w d x\right)^{2 / p} .
$$

Taking the supremum in $\phi$ yields the theorem. Q.E.D.

Remark. This theorem applies even when $T$ is a Hilbert space valued operator.

3. Weighted NORM BOUNDEDNESS OF $C Z_{\varepsilon}^{2}\left(R^{1} \times \cdots \times R^{1}\right)$

3.1. The class $C Z_{\varepsilon}^{2}\left(R^{1} \times \cdots \times R^{1}\right)$. We are going to give the definition of $C Z_{\varepsilon}^{2}\left(R^{1} \times \cdots \times R^{1}\right)$ inductively, beginning with $C Z_{\varepsilon}^{2}\left(R^{1}\right)$. We say $T \in C Z_{\varepsilon}^{2}\left(R^{1}\right)$ if $T$ is a linear operator bounded on $L^{2}\left(R^{1}\right)$ and has the following properties:

(1) there exists a kernel $k(x, y)$ defined on $\left(R^{1} \times R^{1}\right) \backslash \Delta$ where $\Delta=\{(x, y) \mid$ $x=y\}$, and such that if $f, g \in C_{c}^{\infty}\left(R^{1}\right)$ have disjoint supports, then

$$
\int g(x) T(f)(x) d x=\iint g(x) k(x, y) f(y) d x d y .
$$

(2) The kernel $k(x, y)$, in addition, satisfies the condition

$$
\left(\int_{|x-y|>y\left|x-x^{\prime}\right|}\left|k(x, y)-k\left(x^{\prime}, y\right)\right|^{2} d y\right)^{1 / 2} \leq C \gamma^{-(1 / 2+\varepsilon)}\left|x-x^{\prime}\right|^{-1 / 2}, \quad \gamma \geq 2 \text {. }
$$


We define a norm on this class $C Z_{\varepsilon}^{2}\left(R^{1}\right)$ by

$$
\|T\|_{C Z}=\|T\|_{2,2}+\inf _{C \text { in }(2)} C .
$$

For $T \in C Z_{\varepsilon}^{2}\left(R^{1} \times R^{1}\right)$, we require $T$ to be a bounded linear operator on $L^{2}\left(R^{2}\right)$ with

$\left(1^{\prime}\right)$ for each $\left(x_{1}, y_{1}\right),\left(x_{2}, y_{2}\right) \in R^{1} \times R^{1}$, we have operators $k^{1}\left(x_{1}, y_{1}\right)$, $k^{2}\left(x_{2}, y_{2}\right)$ belonging in $C Z_{\varepsilon}^{2}\left(R^{1}\right)$, such that if $f_{i}, g_{i} \in C_{c}^{\infty}\left(R^{1}\right), i=1,2$, then

$$
\begin{aligned}
& \iint g_{1}\left(x_{1}\right) g_{2}\left(x_{2}\right) T\left(f_{1} f_{2}\right)\left(x_{1}, x_{2}\right) d x_{1} d x_{2} \\
& \quad=\iint g_{1}\left(x_{1}\right)\left\langle g_{2}, k^{1}\left(x_{1}, y_{1}\right)\left(f_{2}\right)\right\rangle f_{1}\left(y_{1}\right) d x_{1} d y_{1}
\end{aligned}
$$

whenever $g_{1}, f_{1}$ have disjoint supports. And the dual condition

$$
\begin{aligned}
\iint g_{1}\left(x_{1}\right) g_{2}\left(x_{2}\right) T\left(f_{1} f_{2}\right)\left(x_{1}, x_{2}\right) d x_{1} d x_{2} \\
\quad=\iint g_{2}\left(x_{2}\right)\left\langle g_{1}, k^{2}\left(x_{2}, y_{2}\right)\left(f_{1}\right)\right\rangle f_{2}\left(y_{2}\right) d x_{2} d y_{2}
\end{aligned}
$$

holds whenever $g_{2}, f_{2}$ have disjoint supports.

$\left(2^{\prime}\right)$ The operators $k^{1}\left(x_{1}, y_{1}\right), k^{2}\left(x_{2}, y_{2}\right)$ satisfy

$$
\begin{gathered}
\left(\int_{\left|x_{i}-y_{i}\right|>\gamma\left|x_{i}-x_{i}^{\prime}\right|}|| k^{i}\left(x_{i}, y_{i}\right)-k^{i}\left(x_{i}^{\prime}, y_{i}\right) \|_{C Z}^{2} d y_{i}\right)^{1 / 2} \\
\leq C_{i} \gamma^{-(1 / 2+\varepsilon)}\left|x_{i}-x_{i}^{\prime}\right|^{-1 / 2}, \quad \gamma \geq 2,
\end{gathered}
$$

for $i=1,2$. We define a norm on $T$ by

$$
\|T\|_{C Z}=\|T\|_{2,2}+\sum_{i=1}^{2} \inf _{C_{i} \text { in }\left(2^{\prime}\right)} C_{i} .
$$

The class $C Z_{\varepsilon}^{2}\left(R^{1} \times \cdots \times R^{1}\right)$ is then defined inductively. (See [2].)

We remark here that $C Z_{\varepsilon}^{2}\left(R^{1}\right)$ includes the classical Calderon-Zygmund operator on $R^{1}$, that is, those linear operators bounded on $L^{2}\left(R^{1}\right)$ with kernels $k(x, y)$ satisfying

$$
\begin{gathered}
|k(x, y)| \leq C /|x-y| \\
\left|k(x, y)-k^{\prime}\left(x^{\prime}, y\right)\right| \leq C \frac{\left|x-x^{\prime}\right|^{\delta}}{|x-y|^{1+\delta}}, \quad 2\left|x-x^{\prime}\right| \leq|x-y|, \quad \delta>0 .
\end{gathered}
$$

In fact the product domain generalization of these classical Calderon-Zygmund operators (see Journé [2]) are in $C Z_{\varepsilon}^{2}\left(R^{1} \times \cdots \times R^{1}\right)$. 
3.2. Main estimate for operators in $C Z_{\varepsilon}^{2}\left(R^{1} \times \cdots \times R^{1}\right)$.

Theorem. If $T \in C Z_{\varepsilon}^{2}\left(R^{1} \times \cdots \times R^{1}\right)$ then $T$ is bounded on $L^{p}(\omega)$ for $2<$ $p<\infty, \omega \in A_{p / 2}\left(R^{1} \times \cdots \times R^{1}\right)$.

Proof. We only need to show the main estimate

$$
\int_{\bar{\Omega}}\left|Q_{t}(T f)(x)\right|^{2} d x \frac{d t}{t} \leq C\|T\|_{C Z}^{2} \int_{\Omega} M\left(f^{2}\right)(x) d x .
$$

We are going to use a method composed of techniques developed in [2] and [3]. We first chop $f$ into an inside and outside part with the outside part supported far away from $\Omega$, that is,

$$
f(z)=f \chi_{\Omega_{n+3}}(z)+f \chi_{\Omega_{n+3}^{c}}(z)=f_{I}+f_{0} .
$$

For $f_{I}$, we use the $L^{2}$ boundedness of $T$ and Plancherel equality, so that

$$
\begin{aligned}
\int_{\bar{\Omega}}\left|Q_{t} T\left(f_{I}\right)(x)\right|^{2} d x \frac{d t}{t} & \leq \int_{\bar{R}^{n}}\left|Q_{t} T\left(f_{I}\right)(x)\right|^{2} d x \frac{d t}{t} \\
& =\int_{R^{n}} \prod_{i=1}^{n} \int_{0}^{\infty}\left|\widehat{\psi}\left(t_{i} \xi_{i}\right)\right|^{2}\left|T\left(f_{I}\right)^{-}(\xi)\right|^{2} \frac{d t_{i}}{t_{i}} d \xi \\
& =\left(\int_{0}^{\infty}\left|\widehat{\psi}\left(t_{1}\right)\right|^{2} \frac{d t_{1}}{t_{1}}\right)^{n} \int_{R^{n}}\left|T\left(f_{I}\right)(x)\right|^{2} d x \\
& \leq C\|T\|_{C Z}^{2} \int_{\Omega_{n+3}} f^{2}(x) d x \leq C\|T\|_{C Z}^{2} \int_{\Omega} M\left(f^{2}\right)(x) d x .
\end{aligned}
$$

The last inequality is due to Lemma 5 .

For the term involving $f_{0}$, we need to decompose further. We start by decomposing $\bar{\Omega}$, the region of integration. For each $(x, t) \in \bar{\Omega}$, we have a rectangle $R_{t}(x) \subset \Omega$. To this rectangle we associate a dyadic rectangle $R$ in the manner indicated in $\S 1$. We also have $4 R \supset R_{t}(x)$ and $2 R_{t}(x) \supset R$, and thus, as a consequence, $R \subset \Omega_{1}$. We enlarge the $n$th side of $R$ so that $R \in M^{n}\left(\Omega_{1}\right)$. Thus we have

$$
\bar{\Omega} \subset \bigcup_{R \in M^{n}\left(\Omega_{1}\right)} \overline{4 R} .
$$

For each $R=I_{1} \times I_{2} \times \cdots \times I_{n}$ in the above, we have $\widehat{I}_{1} \times I_{2} \times \cdots \times I_{n} \in M^{\prime}\left(\Omega_{2}\right)$, $\widehat{I}_{1} \times \widehat{I}_{2} \times I_{3} \times \cdots \times I_{n} \in M^{2}\left(\Omega_{3}\right)$, etc. as in Definition 2 of $\S 1$. Now we introduce some notation. We denote $S$ as a subset of $\{1,2, \ldots, n\}$ and $S^{n}$ as the collection of all nonempty subsets of $\{1,2, \ldots, n\}$. And as in [2], we have, writing $S=\left\{s_{1}, s_{2}, \ldots, s_{k}\right\}$ with $s_{1}<s_{2}<\cdots<s_{k}$ the following: for each $(x, t) \in \bar{\Omega}$, we decompose $f_{0}$ in the expression $Q_{t} T\left(f_{0}\right)$ as

$$
f_{0}(z)=\sum_{S \in S^{n}} f_{0}(z)(-1)^{k+1} \chi_{\left(4 \hat{i}_{s_{1}}\right)^{c}}(z) \cdots \chi_{\left(4 \hat{i}_{s_{k}}\right)^{c}}(z) \text {. }
$$


Thus we only need to show for one of these terms above,

$$
\int_{\bar{\Omega}}\left|Q_{t} T\left(f_{0} \prod_{v=1}^{k} \chi_{\left(4 \hat{s}_{s_{v}}\right)^{c}}\right)(x)\right|^{2} d x \frac{d t}{t} \leq C\|T\|_{C Z}^{2} \int_{\Omega} M\left(f^{2}\right)(x) d x
$$

where the intervals $\widehat{I}_{s_{v}}$ depend on the value of $(x, t)$.

To show (1), let us first work for the case $n=1$. We have

$$
\int_{\bar{\Omega}}\left|Q_{t} T\left(f_{0} \chi_{(4 \hat{I})}\right)(x)\right|^{2} d x \frac{d t}{t} \leq \sum_{I \in M^{\prime}\left(\Omega_{1}\right)} \int_{\overline{4 \bar{I}}}\left|Q_{t} T\left(f_{0} \chi_{(4 \hat{I})^{c}}\right)(x)\right|^{2} d x \frac{d t}{t},
$$

where on the right side we lumped all those $(x, t)$ having the same $\widehat{I}$ (these $(x, t)$ must be necessarily in $\overline{4 I})$. Next we write $Q_{t} T\left(f_{0} \chi\right)$ as

$$
\int \psi_{t}(x-y) \sum_{l=1}^{\infty} \int_{|z-x|^{\sim} 2^{l}|I|}(k(y, z)-k(x, z)) f_{0} \chi_{(4 \hat{I})^{c}}(z) d z d y,
$$

where $|x-z|^{\sim} 2^{l}|I|$ means $2^{l}|I| \leq|x-z| \leq 2^{l+1}|I|$. Applying Hölder's inequality, $Q_{t} T\left(f_{0} \chi\right)$ is less than

$$
\begin{aligned}
& C\|T\|_{C Z} \frac{1}{t} \int_{|x-y|<t}|I|^{-\varepsilon}|x-y|^{\varepsilon} \sum_{l=1}^{\infty} 2^{-l \varepsilon}\left[\frac{1}{2^{l}|I|} \int_{|x-z|^{\sim} 2^{l}|I|}|f(z)|^{2} d z\right]^{1 / 2} d y \\
& \leq C\|T\|_{C Z} t^{\varepsilon} \inf _{x \in I} M\left(f^{2}\right)^{1 / 2}(x)|I|^{-\varepsilon} .
\end{aligned}
$$

Substituting the above into the corresponding term in (2), we get

$$
\begin{aligned}
\int_{\bar{\Omega}}\left|Q_{t} T\left(f_{0} \chi\right)\right|^{2}(x) d x \frac{d t}{t} \\
\leq C\|T\|_{C Z}^{2} \sum_{I \in M^{\prime}\left(\Omega_{1}\right)}|I|^{-2 \varepsilon} \int_{4 I} \int_{0}^{2|I|} t^{2 \varepsilon} \frac{d t}{t} d x \inf _{x \in I} M\left(f^{2}\right)(x) \\
\leq C\|T\|_{C Z}^{2} \sum \int_{4 I} M\left(f^{2}\right)(x) d x \\
\leq C\|T\|_{C Z}^{2} \sum \int_{I} M\left(f^{2}\right)(x) d x \quad(\text { Lemma 5) } \\
\leq C\|T\|_{C Z}^{2} \int_{\Omega} M\left(f^{2}\right)(x) d x .
\end{aligned}
$$

The last inequality is a result of the fact that on $R^{1}$, the $I$ in $M^{1}\left(\Omega_{1}\right)$ are disjoint (a fact not true for higher dimensions).

To prove the rest, we use induction on $n$. However, as in [2], we shall need more definitions and a lemma. Let $T \in C Z_{\varepsilon}^{2}$. We denote the operator $\left[Q_{t_{v}} T\right]_{x_{n}, z_{v}}$ acting on the variables $\left(z_{1}, \ldots, z_{v-1}, z_{v+1}, \ldots, z_{n}\right)$ by

$$
\left[Q_{t_{v}} T\right]_{x_{v}, z_{v}}=Q_{t_{v}}\left(k^{4}\left(\cdot, z_{v}\right)-k^{\prime \prime}\left(x_{v}, z_{v}\right)\right) .
$$


Lemma A. If $T \in C Z_{\varepsilon}^{2}\left(R^{n}\right)$, then $\left[Q_{t_{v}} T\right]_{x_{v}, z_{v}} \in C Z_{\varepsilon}^{2}\left(R^{n-1}\right)$, and for an interval $\widehat{I}_{v}$, and a positive integer $l$, we have

$$
\left[\int_{\left|x_{v}-z_{v}\right| 2^{\prime}\left|\hat{I}_{v}\right|}\left\|\left[Q_{t_{v}} T\right]_{x_{v}, z_{v}}\right\|_{C Z}^{2} d z_{v}\right]^{1 / 2} \leq C\|T\|_{C Z}\left(2^{l}\left|\widehat{I}_{v}\right|\right)^{-1 / 2-\varepsilon} t_{v}^{\varepsilon} .
$$

Proof. We write

$$
\left[Q_{t_{v}} T\right]_{x_{v}, z_{v}}=\int \psi_{t_{v}}\left(x_{v}-y_{v}\right)\left(k^{v}\left(y_{v}, z_{v}\right)-k^{v}\left(x_{v}, z_{v}\right)\right) d y_{v} .
$$

Then

$$
\left\|\left[Q_{t_{v}} T\right]_{x_{v}, z_{v}}\right\|_{C Z} \leq \int_{\left|x_{v}-y_{v}\right|}<t_{v} \frac{1}{t_{v}}\left\|k^{v}\left(y_{v}, z_{v}\right)-k^{v}\left(x_{v}, z_{v}\right)\right\|_{C Z} d y_{v} .
$$

Applying Minkowski's integral inequality, Fubini's theorem, and the fact that $T \in C Z_{\varepsilon}^{2}$, we have the conclusion of Lemma A. Q.E.D.

Now we assume (1) is true up to $n-1$, and as a consequence, the main estimate is also verified up to $n-1$. We remind the readers that the indices $\left\{s_{1}, \ldots, s_{k}\right\}$ are fixed throughout this proof, but the intervals $\left\{I_{s_{1}}, \ldots, I_{s_{k}}\right\}$ depend on the point $(x, t)$. For $n$ we have, from earlier pages, the following:

(a) if $(x, t) \in \bar{\Omega}$, then $x \in R_{t}(x) \subset 4 R=4 I_{1} \times \cdots \times 4 I_{n}$ for some $R \in$ $M^{n}\left(\Omega_{1}\right)$,

(b) $\bar{\Omega} \subset \bigcup_{R \in M^{n}\left(\Omega_{1}\right)} \overline{4 R}$,

(c) for each $R=I_{1} \times \cdots \times I_{n}$ from above, let us write $\widehat{R}=\widehat{I}_{1} \times \cdots \times \widehat{I}_{s_{1}-1} \times$ $I_{s_{1}} \times \cdots \times I_{n}$.

We want to decompose $\bar{\Omega}$ into sets so that every $(x, t)$ belonging to a particular set has the same $\widehat{I}_{s_{1}}$ interval. Since for each $(x, t) \in \bar{\Omega}, R_{t}(x)$ is contained in a $4 \widehat{R}, \widehat{R}$ as in (c) above, we group $\widehat{R}$ according to the ratio $\left|\widehat{I}_{s_{1}}\right| /\left|I_{s_{1}}\right|=2^{m}$. For each group corresponding to a fixed $m$, we further sort out $\widehat{R}$ according to $i, j$ in the expression $\left|I_{s_{1}}\right|=2^{i+j(m+1)}, 1 \leq i \leq m+1,-\infty<j<+\infty$. Letting

$$
\begin{aligned}
A_{I, m}=\bigcup\left\{\hat{I}_{1} \times \cdots \times \widehat{I}_{s_{1}-1} \times\right. & I_{s_{1}+1} \times \cdots \times I_{n} \\
& \left.=\widehat{R}^{\prime} \mid \widehat{R}=I_{s_{1}} \times \widehat{R} \in M^{s_{1}-1}\left(\Omega_{s_{1}}\right), \gamma_{s_{1}}(\widehat{R})=2^{m}\right\},
\end{aligned}
$$

$4 A_{I, m}=\bigcup\left\{4 \widehat{R}^{\prime} \mid \widehat{R}^{\prime}\right.$ are rectangles that appear in $\left.A_{I, m}\right\}$,

$$
\overline{4 A}_{I, m}=\bigcup\left\{\overline{4 \widehat{R}^{\prime}} \mid \hat{R}^{\prime} \text { as in } 4 A_{I, m}\right\},
$$

we split the integral on the left side of (1) so that it is dominated by

$$
\sum_{m=1}^{\infty} \sum_{i=1}^{m+1} \sum_{j=-\infty}^{+\infty} \sum^{\prime} \int_{\overline{4}_{s_{1}} \times \overline{4 A}_{l, m}}\left|Q_{t} T\left(f_{0} \prod_{v=1}^{k} \chi_{\left(4 \hat{I}_{s_{v}}\right)^{c}}\right)(x)\right|^{2} d x \frac{d t}{t}
$$


where $\sum^{\prime}$ extends over all dyadic intervals $I_{s_{1}}$ of length $2^{i+j(m+1)}$. This corresponds to (2) in the $n=1$ case. Since the $Q_{t}$ operator is a convolution with a smooth bump function $\psi, \int_{\psi}=0$, we write $Q_{t} T\left(f_{0} \pi \chi\right)$ in (3) as

$$
\left.\sum_{l=1}^{\infty} \int_{\left|x_{s_{1}}-z_{s_{1}}, \sim 2^{\prime}\right| \hat{I}_{s_{1}} \mid} Q_{t^{\prime}}\left[Q_{s_{s_{1}}} T\right]_{s_{s_{1}}, z_{s_{1}}}\left(f_{0}\left(z_{s_{1}}, \cdot\right) \chi_{\left(4 \hat{I}_{s_{v}}\right.}\right)^{c} \cdots\right)\left(x^{\prime}\right) d z_{s_{1}},
$$

where $x=\left(x_{s_{1}}, x^{\prime}\right), t=\left(t_{s_{1}}, t^{\prime}\right)$. This corresponds to (2.1). Thus (3) is dominated by

$$
\begin{aligned}
& \sum \int_{\overline{4 I}}\left\{\sum_{l=1}^{\infty} \int_{\left|x_{s_{1}}-z_{s_{1}} \sim 2^{\prime}\right| \hat{I}_{s_{1} \mid} \mid}\right. \\
& \times\left(\int_{\overline{4 A_{l, m}}}\left|Q_{t^{\prime}}\left[Q_{t_{s_{1}}} T\right]_{{x_{s_{1}}}_{1}, z_{s_{1}}}\left(f_{0} \prod_{v=2}^{k} \chi_{\left(4 \hat{s}_{s_{v}}\right)^{c}}\right)(x)\right|^{2}\right. \\
&\left.\left.\times 8 \frac{d x^{\prime} d t^{\prime}}{t^{\prime}}\right)^{1 / 2} d z_{s_{1}}\right\}^{2} d x_{s_{1}} \frac{d t_{s_{1}}}{t_{s_{1}}}
\end{aligned}
$$

and the induction hypothesis says the above is dominated by

$$
\begin{aligned}
\sum \int_{\overline{4 I_{s_{1}}}}\left\{\sum_{l=1}^{\infty} \int_{\left|x_{s_{1}}-z_{s_{1}} \sim 2^{l}\right| \hat{I}_{s_{1} \mid}}\left\|\left[Q_{t_{s_{1}}} T\right]_{{s_{s_{1}}}_{s_{1}}, z_{s_{1}}}\right\|_{c z}\right. \\
\left.\times \int_{4 A_{l, m}}\left(M\left(f^{2}\right)\left(z_{s_{1}}, x^{\prime}\right) d x^{\prime}\right)^{1 / 2} d z_{s_{1}}\right\}^{2} \frac{d x_{s_{1}} d t_{s_{1}}}{t_{s_{1}}} .
\end{aligned}
$$

Applying Lemma A with Hölder's inequality to the terms inside $\{\cdots\}$ and using

$$
4 A_{I, m} \subset \tilde{A}_{I, m} \subset{\widetilde{E_{I_{s_{1}}} \backslash E_{I_{s_{1}}^{\prime}}}}_{(\text {Lemma 4) }}
$$

where

$$
E_{I_{s_{1}}}=\bigcup\left\{R^{\prime} \mid R^{\prime} \text { rectangles in } R^{n-1} \text { such that } I_{s_{1}} \times R^{\prime} \subset \Omega_{s_{1}}\right\},
$$

we get the above dominated by

$$
\begin{aligned}
& C\|T\|_{C Z}^{2} \sum \int_{4 I_{s_{1}}} \int_{0}^{8\left|I_{s_{1}}\right|}\left|\widehat{I}_{s_{1}}\right|^{-2 \varepsilon} t_{s_{1}}^{2 \varepsilon} d x_{s_{1}} \frac{d t_{1}}{t_{1}} \inf _{x_{s_{1}} \in I_{s_{1}}} \int_{E_{I_{s_{1}}} \backslash E_{I_{s_{1}}}} M\left(f^{2}\right)\left(x_{s_{1}}, x^{\prime}\right) d x^{\prime} \\
& \leq C\|T\|_{C Z}^{2} \sum_{m=1}^{\infty}\left(\frac{\mid I_{s_{1}}}{\left|\widehat{I}_{s_{1}}\right|} \sum^{m+1} \sum_{i=1}^{+\infty} \sum_{j=-\infty}^{\prime} \int_{I_{s_{1}} \times E_{I_{s_{1}}} \backslash E_{I_{s_{1}}}} M\left(f^{2}\right)(x) d x\right. \\
& \quad \leq C\|T\|_{C Z}^{2} \int_{\Omega} M\left(f^{2}\right)(x) d x .
\end{aligned}
$$

The last inequality follows from the same reasoning as in Journés covering lemma. Q.E.D.

After this paper was written, we were informed that R. Fefferman, in [7], had extended (for the operators on the bidisc) the result to the full range of weights, 
i.e., $T: L^{p}(w) \rightarrow L^{p}(x)$ for $w \in A_{p}\left(R^{\prime} \times R^{\prime}\right), 1<p<\infty$. His technique could also be adapted to our case to yield the same result for $w \in A_{p}\left(R^{\prime} \times \cdots \times R^{\prime}\right)$. The technique is based on the result for the "half" weighted case as we have here. Details will appear in a later paper.

\section{The LitTlewood-PALEy TheOREM}

Theorem. If $W=\left\{R_{l}\right\}$ is a collection of disjoint rectangles with sides parallel to the coordinate axes, and $\left(S_{R_{l}} f\right)^{\wedge}(\xi)=\chi_{R_{l}}(\xi) \widehat{f}(\xi)$ then the operator

$$
\Delta f(x)=\left(\sum_{R_{l} \in W}\left|S_{R_{l}}(f)(x)\right|^{2}\right)^{1 / 2}
$$

is bounded on $L^{p}(w)$ for $2<p<\infty, w \in A_{p / 2}\left(R^{1} \times \cdots \times R^{1}\right)$.

4.1. Reductions. Following Rubio de Francia [1], the theorem can be reduced to the special case when the collection of sets $W$ satisfies the well-distributed condition

$$
\sum_{R_{l} \in W} \chi_{16 R_{l}(\xi)} \leq C
$$

In addition we will treat $W$ to be a finite collection, since a limiting argument will yield the general case.

We dyadicize the rectangles in $W$ according to the process indicated in the preliminary definitions. Thus we associate $R_{l} \in W$ with a dyadic rectangle $R_{l}^{d}$ with $2 R_{l} \supset R_{l}^{d}, 4 R_{l}^{d} \supset R_{l}$. We know that for $1<p<\infty, w \in A_{p}$,

$$
\left\|\left(\sum_{R_{l} \in W}\left(S_{R_{l}} f_{l}\right)^{2}\right)^{1 / 2}\right\|_{L^{p}(w)} \leq\left\|\left(\sum_{R_{l} \in W} f_{l}^{2}\right)^{1 / 2}\right\|_{L^{p}(w)}
$$

(this is true for $p=2, w \in A_{2}$; then apply the extrapolation of weights [5]). We let $f_{l}=S_{4 R_{l}^{d}}(f) .2 R_{l} \supset R_{l}^{d}$ and the fact that $W$ is well-distributed imply that each $R_{l}^{d}$ can be associated with at most a fixed number of $R_{l}$. Thus we may assume that each $R_{l}^{d}$ is distinct and

$$
\sum_{l} \chi_{2\left(4 R_{l}^{d}\right)}(\xi) \leq C .
$$

This means we can treat $W$ as if composed of these $4 R_{l}^{d}$. For simplicity, we represent $4 R_{l}^{d} \in W$ as a point in the lattice $\left(Z_{+}^{2}\right)^{n}=\left(k_{1}, j_{1}, \ldots, k_{n}, j_{n}\right)$ by the following [1]. For each $1 \leq i \leq n$, let

$$
\left\{I_{i}\right\}=\left\{I_{i} \mid I_{i}=i \text { th side of } R_{l}^{d}, 4 R_{l}^{d} \in W\right\} .
$$

If $\left|I_{i}\right|=2^{k}$, we label $I_{i}$ as $I_{k_{i}}$. Since there are at most countably many such $I_{k_{i}}$, we label each as $I_{k_{i}}^{j_{i}}, 1 \leq j_{i}<\infty$. Thus each $4 R_{l}^{d} \in W$ can be written as 
$4 R_{k}^{j}=4 I_{k_{1}}^{j_{1}} \times \cdots \times 4 I_{k_{n}}^{j_{n}}$ where $j=\left(j_{1}, \ldots, j_{n}\right), k=\left(k_{1}, \ldots, k_{n}\right)$. We define a set of integers $n_{k}^{j}=\left(n_{k_{1}}^{j_{1}}, \ldots, n_{k_{n}}^{j_{n}}\right)$ to be such that $n_{k_{i}}^{j_{i}} 2^{k_{i}} \in I_{k_{i}}^{j_{i}}$. Here we remark that the only reason we went through this dyadicizing process is to get the fact that whenever $n_{k_{i}}^{j_{i}} \neq n_{k_{i}}^{j_{i}^{\prime}}$ then $j_{i} \neq j_{i}^{\prime}$, a fact we will need.

Next we smooth out our operator in the same manner as [1]. We briefly state it here. We take $\phi \in C^{\infty}\left(R^{1}\right)$ such that $\widehat{\phi}(\xi)=1$ for $|\xi|<2.5$ and $\widehat{\phi}(\xi)=0$ when $|\xi|>3$. Then if

$$
\phi_{k}^{j}(x)=\prod_{i=1}^{n} 2^{k_{i}} \phi\left(2^{k_{i}} x_{i}\right) \exp \left(2 \pi i n_{k_{i}}^{j_{1}} 2^{k_{i}} x_{i}\right)=\prod_{i=1}^{n} \phi_{k_{i}}^{j_{i}}\left(x_{i}\right)
$$

we have

$$
\widehat{\phi}_{k}^{j}(\xi)= \begin{cases}1 & \text { if } \xi \in 4 R_{k}^{j}, \\ 0 & \text { if } \xi \text { is outside } 2\left(4 R_{k}^{j}\right) .\end{cases}
$$

Then the weighted inequality problem turns into (by extrapolation of weights) the weighted problem for the operator

$$
G(f)=\left(\sum_{(k, j) \in W}\left|\Phi_{k}^{j}(f)\right|^{2}\right)^{1 / 2}
$$

where $\Phi_{k}^{j}$ is the operator with kernel

$$
k_{k}^{j}(x, y)=\prod_{i=1}^{n} 2^{k_{i}} \phi\left(2^{k_{i}}\left(x_{i}-y_{i}\right)\right) \exp \left(2 \pi i n_{k_{i}}^{j_{i}} 2^{k_{i}} y_{i}\right) \text {. }
$$

We have trivially the boundedness on $L_{2}$ of $G(f)$ by the well-distributed property of $W$.

4.2. Proof of the main estimate. The theorem follows if we prove the main estimate for $G$ (we are abusing our notation here, actually we treat $G$ as taking $f$ into the $l^{2}$ vector-space valued function $\left.\left\{\Phi_{k}^{j}(f)\right\}\right)$

$$
\int_{\bar{\Omega}} \sum_{(k, j) \in W}\left|Q_{t} \Phi_{k}^{j}(f)(x)\right|^{2} d x \frac{d t}{t} \leq C \int_{\Omega} M\left(f^{2}\right) d x .
$$

We shall need the following lemma, the proof of which will be delayed to $\S 4.3$.

Lemma 6. If $R=I_{1} \times \cdots \times I_{n}$ is a rectangle in $R^{n}$ and $I_{1}^{\prime}, \ldots, I_{n}^{\prime}$ are intervals containing $I_{1}, \ldots, I_{n}$ respectively, then for any $\varepsilon>0$

$$
\int_{\bar{R}} \sum_{k, j}\left|Q_{t} \Phi_{k}^{j}\left(f \prod_{i=1}^{n} \chi_{\left(4 I_{i}^{\prime}\right)} c\right)(x)\right|^{2} d x \frac{d t}{t} \leq C_{\varepsilon} \prod_{i=1}^{n}\left(\frac{\left|I_{i}\right|}{\left|I_{i}^{\prime}\right|}\right)^{\varepsilon}|R| \inf _{z \in R} M\left(f^{2}\right)(z) .
$$

Notice that the sum is over all $(k, j)$ and not just those in $W$.

We start again with

$$
f=f \chi_{\Omega_{n+3}}+f \chi_{\Omega_{n+3}^{c}}=f_{I}+f_{0} .
$$


The term corresponding to $f_{I}$ is treated via Plancherel's equality and the $L^{2}$ boundedness of $G$, that is,

$$
\begin{aligned}
\int_{\bar{\Omega}} \sum_{(k, j) \in W}\left|Q_{t} \Phi_{k}^{j}\left(f_{I}\right)(x)\right|^{2} d x \frac{d t}{t} & \leq \int_{\bar{R}^{n}} \sum\left|Q_{t} \Phi_{k}^{j}\left(f_{I}\right)(x)\right|^{2} d x \frac{d t}{t} \\
& \leq C \int_{\Omega_{n+3}} f^{2}(x) d x \leq C \int_{\Omega} M\left(f^{2}\right)(x) d x .
\end{aligned}
$$

We decompose the $f_{0}$ term as in $\S 3.2$. In short, for each $(x, t)$ in $\bar{\Omega}$, we can decompose $f_{0}$ in $Q_{t} \Phi_{k}^{j}\left(f_{0}\right)(x)$ into

$$
f_{0}(z)=\sum_{S \in S^{n}} f_{0}(z)(-1)^{k+1} \chi_{\left(4 \widehat{I}_{s_{1}}\right)^{c}} \cdots \chi_{\left(4 \widehat{I}_{s_{k}}\right)^{c}},
$$

where $S^{n}$ is a collection of all nonempty subsets of $\{1, \ldots, n\}$, and $S=$ $\left\{s_{1}, \ldots, s_{k}\right\}$ with $1 \leq s_{1}<\cdots<s_{k} \leq n$. To prove the estimate, we only need to show the following for one of those terms above:

$$
\int_{\bar{\Omega}} \sum_{(k, j) \in W}\left|Q_{t} \Phi_{k}^{j}\left(f_{0} \prod_{v=1}^{k} \chi_{\left(4 \hat{I}_{s_{v}}\right)^{c}}\right)(x)\right|^{2} d x \frac{d t}{t} \leq C \int_{\Omega} M\left(f^{2}\right) d x .
$$

We decompose $\bar{\Omega}$ in (1) in the same manner as in (3) of $\S 3.2$, so that the left-hand side of (1) is less than

$$
\sum_{m_{1}=1}^{\infty} \sum_{i_{1}=1}^{m_{1}+1} \sum_{j_{1}=-\infty}^{+\infty} \sum^{\prime} \int_{\overline{4 I}_{s_{1}} \times \overline{4 A}_{s_{s_{1}}, m_{1}}} \sum_{(k, j) \in W}\left|Q_{t} \Phi_{k}^{j}\left(f_{0} \prod_{v=1}^{k} \chi_{\left(4 \hat{I}_{s_{v}}\right)^{c}}\right)(x)\right|^{2} d x \frac{d t}{t} .
$$

If we let $\sum_{1}=\sum_{m_{1}=1}^{\infty} \cdots \sum^{\prime}$ in the above, then the above term is dominated by

$$
\begin{aligned}
& \sum_{1} \int_{\overline{4 I}_{s_{1}}} \sum_{k_{1}, j_{1}} \int_{\overline{4 A}_{I_{s_{1}, m_{1}}}} \\
& \times \sum_{\left(k^{\prime}, j^{\prime}\right)}\left|Q_{t^{\prime}} \Phi_{k^{\prime}}^{j^{\prime}}\left[Q_{t_{s_{1}}} \Phi_{k_{s_{1}}}^{j_{s_{1}}}\left(f_{0} \chi_{\left(4 \hat{I}_{s_{1}}\right) c^{c}}\right) \prod_{v=2}^{k} \chi_{\left(4 \hat{I}_{s_{v}}\right)^{c}}\right]\left(x_{s_{1}}, x^{\prime}\right)\right|^{2} d x^{\prime} \frac{d t^{\prime}}{t^{\prime}} d x_{s_{1}} \frac{d t_{s_{1}}}{t_{s_{1}}},
\end{aligned}
$$

where $x=\left(x_{s_{1}}, x^{\prime}\right), t=\left(t_{s_{1}}, t^{\prime}\right), k=\left(k_{s_{1}}, k^{\prime}\right), j=\left(j_{s_{1}}, j^{\prime}\right)$. Let

$$
F_{x_{1}}\left(z^{\prime}\right)=Q_{t_{s_{1}}} \Phi_{k_{s_{1}}}^{j_{s_{1}}}\left(f_{0} \chi_{\left(4 \hat{l}_{s_{1}}\right)^{\prime}}\right)\left(z^{\prime}\right)
$$

We see that the term

$$
\int_{\overline{4 A}_{s_{s_{1}, m_{1}}}} \sum_{k^{\prime}, j^{\prime}}\left|Q_{t^{\prime}} \Phi_{k^{\prime}}^{j^{\prime}}\left(F_{x_{s_{1}}} \prod_{v=2}^{k} \chi_{\left(4 \hat{s}_{s_{v}}\right)^{c}}\right)\left(x^{\prime}\right)\right|^{2} d x^{\prime} \frac{d t^{\prime}}{t^{\prime}}
$$

is the $n-1$ version. Now if the term $\prod_{v=2}^{k} \chi_{\left(4 \hat{I}_{s_{v}}\right)^{c}}=1$ (that is, $k=1$ ), then we can apply the same kind of decomposition as in the beginning of this section, 
that is,

$$
F_{x_{s_{1}}}\left(x^{\prime}\right)=F_{x_{s_{1}}} \chi_{\left(4 A_{I_{s_{1}, m_{1}}}\right)_{n+2}}+F_{x_{s_{1}}} \chi_{\left(4 A_{s_{s_{1}}, m_{1}}\right)_{n+2}^{c}}^{c}=F_{x_{s_{1}} ; I}+F_{x_{s_{2}} ; 0} .
$$

Then use $L^{2}$ boundedness of the operator $G\left(n-1\right.$ version) for $F_{x_{s} ; I}$, and decompose $F_{x_{s_{1}} ; 0}$ as in $(* *)$ for each $\left(x^{\prime}, t^{\prime}\right) \in \overline{4 A}_{I_{s_{1}, m_{1}}}$. If $\prod_{v=2}^{k} \chi_{\left(4 \hat{I}_{s_{v}}\right)^{c}}$ is not equal to $1(k>1)$, then we continue to reduce to a lower dimensional case. Thus we will need to check only the following two cases:

$$
\begin{gathered}
\sum_{1} \cdots \sum_{n} \int_{\overline{4 I}_{1}} \cdots \int_{\overline{4 I}_{n}} \sum_{(k, j) \in W}\left|Q_{t} \Phi_{k}^{j}\left(f_{0} \prod_{v=1}^{n} \chi_{\left(4 \hat{I}_{v}\right)^{c}}\right)(x)\right|^{2} d x \frac{d t}{t} \\
\sum_{1} \cdots \sum_{p} \int_{\overline{4 I}_{s_{1}}} \cdots \int_{\overline{4 I}_{s_{p}}} \int_{4 A_{s_{s_{1}} \cdots I_{s_{p}}}, m_{1}, \ldots, m_{p}} \\
\times \sum_{\substack{k_{s_{1}} \cdots k_{s_{p}} \\
j_{s_{1}} \cdots j_{s_{p}}}}\left|Q_{t^{\prime \prime}} \Phi_{k^{\prime \prime}}^{j^{\prime \prime}}\left(f_{0}\right)\left(x^{\prime \prime}, x^{\prime \prime \prime}\right)\right|^{2} d s^{\prime \prime \prime} \frac{d t^{\prime \prime} d t^{\prime \prime}}{t^{\prime \prime}}
\end{gathered}
$$

where $\left\{s_{1}, \ldots, s_{p}\right\}$ is some subset of $\{1, \ldots, n\}, x^{\prime \prime}=\left(x_{s_{1}}, \ldots, x_{s_{p}}\right), x=$ $\left(x^{\prime \prime}, x^{\prime \prime \prime}\right)$, etc. The case $(2.1)$ is handled by Lemma 6 , that is, $(2.1)$ becomes

$$
\begin{gathered}
\sum_{1} \cdots \sum_{n} \int_{\overline{4 R}} \sum_{(k, j)}\left|Q_{t} \Phi_{k}^{j}\left(f_{0} \prod_{v=1}^{n} \chi_{\left(4 \hat{I}_{v}\right)^{c}}\right)(x)\right|^{2} d x \frac{d t}{t} \\
\leq C \sum_{1} \cdots \sum_{n} \prod_{v=1}^{n}\left(\frac{\left|I_{v}\right|}{\left|\hat{I}_{v}\right|}\right)^{\varepsilon}|R| \inf _{z \in R} M\left(f^{2}\right)(z),
\end{gathered}
$$

which, by Journé's covering lemma, is dominated by

$$
C \int_{\Omega} M\left(f^{2}\right)(x) d x
$$

(2.2) is equal to

$$
\sum_{1} \cdots \sum_{p} \int_{4 A_{I, m}} \int_{\overline{4 R}} \sum_{k_{s_{1}} \cdots k_{s_{p}}}\left|Q_{t^{\prime \prime}} \Phi_{k^{\prime \prime}}^{j^{\prime \prime}}\left(f_{0}\right)\left(x^{\prime \prime}, x^{\prime \prime \prime}\right)\right|^{2} d x^{\prime \prime} \frac{d t^{\prime \prime}}{t^{\prime \prime}} d x^{\prime \prime \prime},
$$

where $R=I_{s_{1}} \times \cdots \times I_{s_{p}}$. Then the inner integral is dominated by (as a consequence of Lemma 6 )

$$
C \sum_{1} \cdots \sum_{p} \prod_{v=1}^{p}\left(\frac{\left|I_{s_{v}}\right|}{\left|\widehat{I}_{s_{v}}\right|}\right)^{\varepsilon}\left|I_{s_{v}}\right| \int_{4 A_{I, m}} \inf _{x^{\prime \prime} \in I_{s_{1}} \times \cdots \times I_{s_{p}}} M\left(f^{2}\right)\left(x^{\prime \prime}, x^{\prime \prime \prime}\right) d x^{\prime \prime \prime} .
$$

Then applying Journé's covering lemma $p$ times, we get the above dominated by

$$
C \int_{\Omega} M\left(f^{2}\right)(x) d x
$$


4.3. Proof of Lemma 6. So we are left with

Lemma 6. If $R=I_{1} \times \cdots \times I_{n}$ in a rectangle in $R^{n}$ and $I_{1}^{\prime}, \ldots, I_{n}^{\prime}$ are intervals containing $I_{1}, \ldots, I_{n}$ respectively, then for any $\varepsilon>0$

$$
\int_{\bar{R}} \sum_{k, j}\left|Q^{t} \Phi_{k}^{j}\left(f \prod_{i=1}^{n} \chi_{\left(4 I_{i}^{\prime}\right)^{c}}\right)(x)\right|^{2} d x \frac{d t}{t} \leq C \prod_{i=1}^{n}\left(\frac{\left|I_{i}\right|}{\left|I_{i}^{\prime}\right|}\right)^{\varepsilon}|R| \inf _{z \in R} M\left(f^{2}\right)(z) .
$$

The sum is over all $(k, j)$ and not just those in $W$.

Proof. We shall prove it for $n=2$. The general case is obvious from this proof. We first claim that for $n=1$ we have (we denote $|x-z|^{\sim} z^{l}\left|I^{\prime}\right|$ as $\left.2^{l}\left|I^{\prime}\right| \leq|x-z| \leq 2 \cdot 2^{l}\left|I^{\prime}\right|\right)$

$$
\sum_{k, j}\left|Q_{t} \Phi_{k}^{j}\left(f \chi_{\left(4 I^{\prime}\right)^{c}}\right)(x)\right|^{2} \leq\left. C t^{\varepsilon}\left|I^{-\varepsilon} \sum_{l=0}^{\infty} 2^{-l \varepsilon / 2} \frac{1}{\left|I^{\prime}\right| 2^{l}} \int_{|x-2|^{\sim} 2^{\prime}\left|I^{\prime}\right|}\right| f(z)\right|^{2} d z .
$$

We linearize the expression. Let $H=\left\{\lambda_{k, j}\right\}$ with norm $\left(\sum_{k, j}\left|\lambda_{k, j}\right|^{2}\right)^{1 / 2}$. For each fixed $(x, t)$ we pick $\left\{\lambda_{k, j}(x, t)\right\}$ with norm 1 such that

$$
\begin{aligned}
\left(\left.\sum_{k, j}|| Q_{t} \Phi_{k}^{j}\left(f \chi_{\left(4 I^{\prime}\right)}\right)(x)\right|^{2}\right)^{1 / 2} & =\sum_{k, j} \lambda_{k, j}(x, t) \mid Q_{t} \Phi_{k}^{j}(f \chi)(x) \\
& =Q_{t}\left(\sum_{k, j} \lambda_{k, j}(x, t) \Phi_{k}^{j}(f \chi)\right)(x) .
\end{aligned}
$$

The latter expression becomes, using $\int \psi=0$,

$$
\int \psi_{t}(x-y) \int\left\{\sum_{k, j}\left(k_{k, j}(y, z)-k_{k, j}(x, z)\right) \lambda_{k, j}(x, t)\right\} f \chi d z d y
$$

which is dominated by (let $K_{\lambda}(y, z)-K_{\lambda}(x, z)$ be the expression inside $\left.\{\cdots\}\right)$

$$
\int_{|x-y|<1} \sum_{l=0}^{\infty} \int_{|x-z|^{\sim} 2^{\prime}\left|I^{\prime}\right|}\left|K_{\lambda}(y, z)-K_{\lambda}(x, z)\right||f(z)| d z \frac{d y}{t}
$$

which is dominated by

$$
\begin{aligned}
\int_{|x-y|<t} \sum_{l=0}^{\infty}\left(\int_{|x-z|^{\sim} 2^{\prime}\left(\left|I^{\prime}\right| /|x-y|\right)|x-y|}\left|K_{\lambda}(y, z)-K_{\lambda}(x, z)\right|^{2} d z\right)^{1 / 2} & \\
& \times\left(\int_{|x-z|^{\prime} 2^{\prime}\left|I^{\prime}\right|}|f(z)|^{2} d z\right)^{1 / 2} \frac{d y}{t} .
\end{aligned}
$$

Now we use a lemma from Rubio de Francia [1, pp. 8-12], which says whenever we have $j \neq j^{\prime}$ then $n_{k}^{j} \neq n_{k}^{j^{\prime}}$. Then

$$
\int_{|x-z|^{\sim} \gamma|x-y|}\left|K_{\lambda}(y, z)-K_{\lambda}(x, z)\right|^{2} d x \leq C \frac{\gamma^{-(1+\varepsilon)}}{|x-y|} .
$$


Applying this fact we get

$$
\begin{aligned}
& \left(\sum_{k, j}\left|Q_{t} \Phi_{k}^{j}\left(f \chi_{\left(4 I^{\prime}\right)^{c}}\right)(x)\right|^{2}\right)^{1 / 2} \\
& \quad \leq C t^{\varepsilon / 2}\left|I^{\prime}\right|^{-\varepsilon / 2} \sum_{l} 2^{-l \varepsilon / 2}\left(\frac{1}{2^{l}\left|I^{\prime}\right|} \int_{|x-z|^{\sim} 2^{l}\left|I^{\prime}\right|}|f(z)|^{2} d z\right)^{1 / 2} \\
& \quad \leq C t^{\varepsilon / 2}\left|I^{\prime}\right|^{-\varepsilon / 2}\left(\sum_{l} 2^{-l \varepsilon / 2} \frac{1}{2^{l}\left|I^{\prime}\right|} \int_{|x-z|^{\sim} 2^{l}\left|I^{\prime}\right|}|f(z)|^{2} d z\right)^{1 / 2} .
\end{aligned}
$$

Now then for the proof of Lemma 6 ,

$$
\begin{aligned}
\int_{\bar{I} \times \bar{J}} & \sum_{k_{2}, j_{1}} \sum_{k_{1}, j_{1}}\left|Q_{t_{1}} \Phi_{k_{1}}^{j_{1}}\left(Q_{t_{2}} \Phi_{k_{2}}^{j_{2}} f \chi_{\left(4 J^{\prime}\right)^{c}} \chi_{\left(4 I^{\prime}\right)^{c}}\right)\left(x_{1}\right)\right|^{2} d x \frac{d t}{t} \\
\leq & C \int_{\bar{I} \times \bar{J}}\left(t_{1}^{\varepsilon}\left|I^{\prime}\right|^{-\varepsilon} t_{2}^{\varepsilon}\left|J^{\prime}\right|^{-\varepsilon} \sum_{l} 2^{-l \varepsilon / 2} \frac{1}{\left|I^{\prime}\right| 2^{l}} \int_{\left|x_{1}-z_{1}\right|^{\sim} 2^{\prime}\left|I^{\prime}\right|}\left|f\left(z_{1}, z_{2}\right)\right|^{2} d z_{2} d z_{1}\right) d x \frac{d t}{t} \\
& \times \sum_{l^{\prime}} 2^{-l^{\prime} \varepsilon / 2} \frac{1}{\left|J^{\prime}\right| 2^{l^{\prime}}} \int_{\left|x_{2}-z_{2}\right|^{\sim} 2^{l^{\prime}\left|J^{\prime}\right|}} \mid t_{2}^{|J|} d t_{2}\left(\left|I^{\prime}\right|\left|J^{\prime}\right|\right)^{-\varepsilon} \inf _{z \in R} M\left(f^{2}\right)(z) \\
\leq & C \int_{I} d x_{1} \int_{0}^{|I|} t_{1}^{\varepsilon-1} d t_{1} \int_{J} d x_{2} \int_{0}^{\mid J-1} t_{2}^{\varepsilon}\left(\frac{|J|}{\left|I^{\prime}\right|}\right)^{\varepsilon}|R| \inf _{z \in R} M\left(f^{2}\right)(z) .
\end{aligned}
$$

And this yields the lemma.

\section{REFERENCES}

1. José L. Rubio de Francia, A Littlewood-Paley inequality for arbitrary intervals, Mittag-Leffler Institute, Report No. 18, 1983.

2. Jean-Lin Journé, Calderon-Zygmund operators on product spaces, Rev. Mat. Iberoamericana 1 (1985), 55-91.

3. Robert Fefferman, Harmonic analysis in product spaces, Ann. of Math. (2) 126 (1987), 109-130.

4. C. Fefferman and E. M. Stein, Some maximal inequalities, Amer. J. Math. 93 (1971), 107-115.

5. José L. Fubio de Francia, Factorization and extrapolation of weights, Bull. Amer. Math. Soc. 7 (1982), 393-396.

6. J. Pipher, Journé's covering lemma and its extension to higher dimensions, Duke J. Math. 53 (1986), 683-690.

7. R. Fefferman, $A_{p}$ weights and singular integrals, Amer. J. Math. 110 (1988), 975-987.

Division of Mathematics, F.A.T., Auburn University, 218 Parker Hall, Auburn, AlABAMA 36849 